\title{
Genesis, Evaluation and Progression of A Breakthrough Discovery to Efficiently Cure Cancer Through Use of Dr. M.S. Reddy's Multiple Mixed Strain Probiotics as Adjuvants Along with the Traditional Cancer Therapies, Through Restoration of Healthy and Balanced Intestinal Microbiota and their Microbiome
}

\author{
Malireddy S Reddy, BVSc (DVM), MS, PhD.* \\ Department of Biopharmacology, International Media and Cultures (IMAC) Inc and ADFAC Labs, Inc., USA
}

*Corresponding author: Malireddy S Reddy, Department of Biopharmacology, International Media and Cultures (IMAC) Inc and ADFAC Labs, Inc., 1280 S. Parker Road, Denver, C0 80231, USA. Email: drreddy@askimac.com

\begin{abstract}
A breakthrough, Multiple Mixed Strain Probiotic Therapy, although originally designed to cure hospital acquired infections, has been discovered serendipitously by Dr. M.S. Reddy to treat cancer with greatest accuracy (60\% as opposed to current $20 \%$ ), with least or no side effects and least or no relapse, when used as an adjuvant therapy along with standard cancer therapies, including immune checkpoint therapy. This discovery can be further refined and improved to treat cancer at 80 to $100 \%$ efficacy to save lives of millions of people in the world by significantly improving immune system through positive manipulation of the human gut microbiota and their microbiome. The results of the clinical trials are outlined along with discussion and conclusion. The references listed have been limited to the latest, significant, and directly applicable research discoveries only.

Keywords: Dr MS Reddy's multiple mixed strain probiotic therapy to treat hospital acquired infections; Nosocomial infections; Dr MS Reddy multiple mixed strain adjuvant cancer therapy; Microbiota; Hospital acquired infections; Immune checkpoint therapy; C Diff; MRSA; Microbiome
\end{abstract}

\section{Introduction}

A brief history on statistics of the cancer episode in the world. The International agency for Research on Cancer (IARC), a division of The World Health Organization (WHO), estimated that the global cancer epidemic rose to 18.1 million new cases and 9.6 million deaths in 2018 alone. The IARC reports 1 in 5 men (20\%) and 1 in 6 women $(16.66 \%)$ worldwide develop cancer during their lifetime. 1 in 8 men (12.5\%) and 1 in 11 women (9.09\%) die from the disease. In addition, every sixth death in the world is due to cancer. Assuming world population is 7 billion and assuming the ratio of male to female is 50:50, 700 million men and 583 million women will develop cancer during their life time and 437 million men and 318 million women will die of cancer. These figures are astronomical, mind blowing, and downright scary, unless something is done to prevent or cure cancer efficiently with 100 percent accuracy, without any relapse.

\section{Materials, Methods, and Discussion}

The question here is, how did Dr. M.S. Reddy's discovery to treat cancer came about serendipitously? In the year 2016, Dr. M.S. Reddy, after working extensively for four decades on probiotics, published a breakthrough research article on the usage of Multiple Mixed Strain Probiotic Therapy to treat or prevent the hospital acquired or nosocomial infections caused by Clostridium difficile (C. diff), and Methicillin Resistant Staphylococcus Aureus (MRSA) [1-4]. He has isolated and selected the strains of Probiotics which are naturally resistant to several antibiotics and sulfonamides to use in this discovery. Dr. M.S. Reddy has studied and confirmed 
through thorough research that, the antibiotic resistance was not coded by the plasmids but by the chromosol genes, thus it is safer to use these Multiple Mixed Strain Probiotics to eliminate the free exchange of plasmids to the pathogenic bacteria [5]. The Multiple Mixed Strain Probiotic Strains belonging to different genera and species (with varied physiological characteristics) were grown separately in selected nutritionally balanced media, not only to maximize their cell numbers and their single cell morphology (as opposed to chain formation), but also to significantly improve the quantity of their end products of growth i.e. immunomodulins, bacteriocins, and specific and nonspecific bio-active peptides etc. Such individually grown single strain probiotics were blended in fixed proportions and frozen using liquid nitrogen, to be used as therapeutic agents administered through oral route [3]. Unlike the popular thinking, the significant feature of the discovery is that the probiotic bacterial cultures alone (without their prior growth end products) did not cure the hospital acquired infections. However, the multiple growth end products of these Multiple Mixed strain Probiotics (immunomodulins) along with the active probiotic bacteria significantly cured the nosocomial infections [6].

It was also determined and confirmed that the multiple antibiotic resistant pathogenic bacteria i.e. C. diff etc. was initially inhibited (in vivo) through the immunodolation exerted by the Multiple Mixed Strain Probiotic growth end products along with the dendritic cells picked up probiotic cell wall antigens. The immunomodulation induced by probiotics was through production of IL-10, retinoic acid, TGF-Beta with resultant well balanced activation of T-reg cells [7]. The excess inflammation caused due to immune stimulation as a response to the pathogenic bacterial peptides of C. diff bacteria, through activation of Th-1, Th-2, Th-17 and excess production of IGA, was reduced predominantly through activation of T-reg cells stimulated by Multiple Mixed Strain Probiotics and their immunomodulins, used in this discovery [7]. As a second step (after establishment of positive immunomodulation in vivo), the live probiotic cells in the intestinal tract lumen started directly inhibiting the pathogenic C diff organisms etc. Thus, this invention is novel in that the primary inhibition of the pathogenic bacteria is indirectly due to the previously produced active immunomodulins of the probiotic bacteria, followed by the secondary direct physical inhibition due to live probiotic bacteria in the Lumen, through nutritional competency, production of organic acids, bacteriocins, and finally through stimulation and maintenance of component balance of other non-pathogenic saprophytic intestinal microbiota [5,7]. The bacteriological analysis of patient's feces, after the multiple probiotic infusion treatment, revealed total absence of pathogenic C. diff organisms, and at the same time significant elevation of intestinal microbiota (in comparison to the beginning of treatment) by two logs (determined by combination of anaerobic as well as aerobic total bacterial counts). In addition, it was proven that the individual strains used in the Multiple Mixed Strain Probiotic Therapy did adhere to the intestinal epithelial cells of the host, which was confirmed by biopsy and subsequent microbiological testing including strain specific phage typing.
While conducting the practical clinical trials using Dr. M.S. Reddy's Multiple Mixed Strain Probiotics to prevent or cure hospital acquired infections, Dr. Reddy came up with another novel breakthrough serendipitous discovery to cure cancer with a significantly greater efficiency, when such Multiple Mixed Strain Probiotic Therapy is used as an adjuvant in combination with standard cancer therapies, including the immune checkpoint therapy. This breakthrough discovery has been published in 2018 [7]. Dr. M.S. Reddy's discovery is termed as “Dr. M.S. Reddy's Multiple Mixed Strain Probiotic Adjuvant Cancer Therapy". This Adjuvant Cancer Therapy establishes immune tolerance and thus helps the primary cancer therapy to be most effective, with least or no side effects. It also significantly reduced the relapse of cancer, which was proven through multiple community based clinical trials. This was accomplished through positive maintenance of the cancer patients gut microbiota and their microbiome, thus establishing the proper immunomodulation which results in significant reduction of immune stimulation and inflammation. This in turn established a positive immune tolerance, which is the prime requisite to synergistically improve the efficiency of the standard cancer therapies. This explains why most of the previous cancer therapies were not effective to cure cancer with greater efficiency (of not more than 20 percent).

The clinical tests (with regard to treatment or prevention of nosocomial infections) clearly proved the following, using the procedure outlined in our earlier publication $[1,5]$. The antibiotic therapy (using vancomycin and bacitracin) alone could not cure the hospital acquired infections; probiotic therapy by itself showed significant progress even after one week treatment followed by total cure by 4 th week; prior administration of probiotics (prior to hospitalization) for a period of 2 weeks, significantly reduced C. diff infection; the subjects who were given preventive probiotics for a period of 3 weeks (prior to hospitalization) did not pick up any C. diff infection, during and after hospitalization; combination of antibiotic and Multiple Mixed Strain Probiotic Therapy cured the C. diff infection in two weeks compared to probiotic therapy alone (which took 4 weeks) and antibiotic therapy alone (which could not cure even after, and beyond 4 weeks of treatment). Although it was a serendipitous discovery, later several planned community based clinical trials conducted using Multiple Mixed Strain Probiotic Therapy as an Adjuvant Cancer Therapy, along with the immune checkpoint therapy and other standard cancer therapies, proved that cancer was cured with $60 \%$ efficiency, as opposed to $20 \%$ by immune checkpoint therapy and other standard therapies alone. Unlike the pharmaceutical cancer drugs, Multiple Mixed Strain Probiotics come under nutritional supplements, as per the Code of Federal Regulations, and thus are highly economical and affordable and can be used without any major governmental regulations [2].

\section{Conclusion}

Dr. Reddy's Multiple Mixed Strain Probiotic Adjuvant Cancer Therapy, proven through extensive basic research and continued 
multiple practical community based clinical trials, found to be the best complementary therapy to cure cancer along with the use of immune checkpoint therapy and other traditional cancer therapies. Ultimately, this could be the answer to prevent or treat cancer with 80 to $100 \%$ accuracy with no immune related side effects and with least relapse. Thus, cancer can be treated using such a complementary adjuvant probiotic therapy, along with any chosen conventional cancer therapy, as a choice therapy than a chance therapy, to save lives of over 9 million people in the world per year. It is the first time in the world, where a missing link in the successful treatment of cancer has been discovered, and immense credit should go to this breakthrough medical discovery. This discovery can be further improved to develop customized treatment by taking into account the genetics of the cancer patients to cure several cancers. Multiple Mixed Strain Probiotic Therapy, coupled with positive nutritional and life style modifications, may also serve as a preventive aid to eliminate or reduce cancer epidemic by maintaining the healthy microbiota and their microbiome.

\section{Acknowledgment:}

I am extremely thankful to all the physicians, clinical laboratory personnel, and patients who have participated in the clinical trials. Sincere thanks and appreciation go to all the staff of International Media and Cultures (IMAC-USA) and ADFAC Labs, Pvt. Ltd., India, for their untiring assistance in research and for running clinical trials on a timely manner throughout this study. Thanks, are also extended to Mrs. Renee Williams for valuable assistance for coding the data and preparing this manuscript.

\section{References}

1. Reddy MS, Reddy DRK (2016) Development of Multiple Mixed Strain Probiotics for "Probiotic therapy" under clinical conditions, to prevent or cure the deadly hospital acquired infections due to Clostridium difficile (C. diff) and Methicillin Resistant Staphylococcus Aureus (MRSA). Int J Pharma Sci Nanotech 9(3): 3256-3281.

2. Reddy MS, Reddy DRK (2016) Isolation and determination of the major principle of causative agent behind the 2016 published breakthrough discovery of Dr. M.S. Reddy's "Multiple Mixed Strain Probiotic Therapy" in successfully treating the lethal hospital acquired infections due to Clostridium difficile (C. diff) and Methicillin Resistant Staphylococcus Aureus (MRSA). Int J Pharma Sci Nanotech 9(6): 3556-3566.

3. Reddy MS, Reddy DRK (2016) Dr MS Reddy's Multiple Mixed Strain Probiotic Therapy. J Pharmaceutics and Nano Technol 4(3): 15-28.

4. Reddy MS, Reddy DRK (2017) An insight into the 2016 Best Medical Award-Winning Breakthrough Microbial and Nanotechnology based discovery of Dr. M.S. Reddy's Multiple Mixed Strain Probiotic Therapy, to successfully treat the Nosocomial infections. Nanotechnology and Nanoscience Journal 1(1): 2-5.

5. Reddy MS (2018) A Multiple Mixed Strain Probiotic Therapy to treat or prevent hospital acquired infections. AAPI (American Association of Physicians of Indian Origin) journal. Spring 38.

6. Reddy MS (2018) Immunomodulatory effect of “Dr. M.S. Reddy's Multiple Mixed Strain Probiotic Therapy" to cure or prevent hospital acquired nosocomial infections due to Clostridium difficile (C. diff), other pathogenic bacteria, and autoimmune diseases. Int J Pharma Sci Nanotech 11(1): 3937-3949.

7. Reddy MS (2018) Dr. M.S. Reddy's Multiple Mixed Strain Probiotic Adjuvant Cancer Therapy, to complement immune checkpoint therapy and other traditional cancer therapies, with least autoimmune side effects through eco-balance of human microbiome Int J. Pharma Sci Nanotech 11(6): 4295-4317.

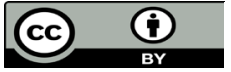

This work is licensed under Creative Commons Attribution 4.0 License

To Submit Your Article Click Here:

Submit Article

DOI: $10.32474 /$ LOJPCR.2019.01.000121

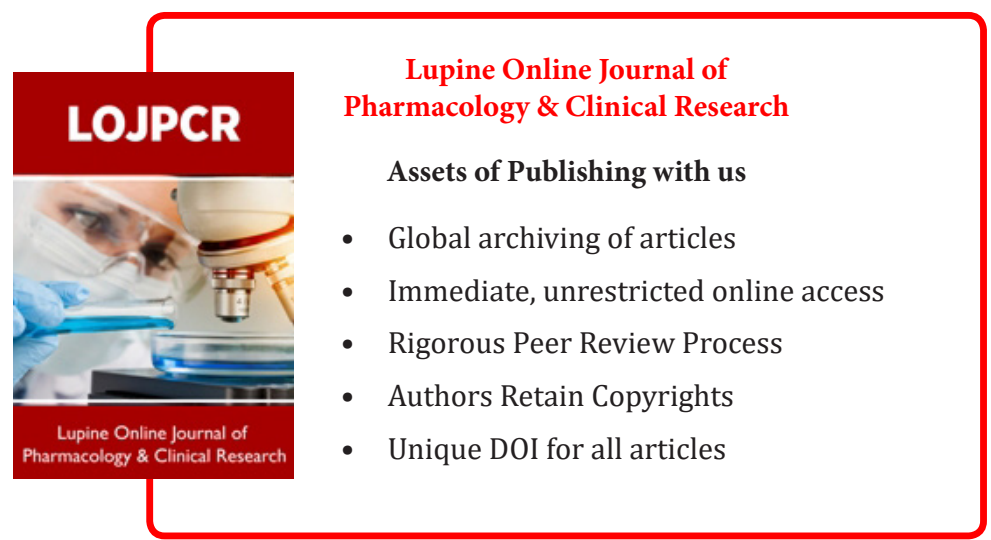

Citation: Malireddy S Reddy. Genesis, Evaluation and Progression of A Breakthrough Discovery to Efficiently Cure Cancer Through Use of Dr. M.S. 